\title{
Monotone Drawings of Graphs with Fixed Embedding *
}

\author{
Patrizio Angelini ${ }^{1}$, Walter Didimo ${ }^{2}$, Stephen Kobourov ${ }^{3}$, Tamara Mchedlidze ${ }^{4}$, \\ Vincenzo Roselli ${ }^{1}$, Antonios Symvonis ${ }^{4}$, and Stephen Wismath ${ }^{5}$ \\ ${ }^{1}$ Università Roma Tre, Italy \\ 2 Università degli Studi di Perugia, Italy \\ ${ }^{3}$ University of Arizona, USA \\ 4 National Technical University of Athens, Greece \\ ${ }^{5}$ University of Lethbridge, Canada
}

\begin{abstract}
A drawing of a graph is a monotone drawing if for every pair of vertices $u$ and $v$, there is a path drawn from $u$ to $v$ that is monotone in some direction. In this paper we investigate planar monotone drawings in the fixed embedding setting, i.e., a planar embedding of the graph is given as part of the input that must be preserved by the drawing algorithm. In this setting we prove that every planar graph on $n$ vertices admits a planar monotone drawing with at most two bends per edge and with at most $4 n-10$ bends in total; such a drawing can be computed in linear time and in polynomial area. We also show that two bends per edge are sometimes necessary on a linear number of edges of the graph. Furthermore, we investigate subclasses of planar graphs that can be realized as embedding-preserving monotone drawings with straight-line edges, and we show that biconnected embedded planar graphs and outerplane graphs always admit such drawings, which can be computed in linear time.
\end{abstract}

\section{Introduction}

A drawing of a graph is a monotone drawing if for every pair of vertices $u$ and $v$, there is a path drawn from $u$ to $v$ that is monotone in some direction. In other words, a drawing is monotone if and only if, for any given direction $d$ (e.g., from left to right) for each pair of vertices $u$ and $v$, there exists a suitable rotation of the drawing for which a path from $u$ to $v$ becomes monotone in the direction $d$.

Monotone drawings have been recently introduced [1] as a new visualization paradigm, which is well motivated by human subject experiments by Huang and Eades [10] who showed that the "geodesic tendency" (paths follow a given direction) is important in comprehending the underlying graph. Monotone drawings are related to well-studied drawing conventions, such as upward drawings [5, 8], greedy drawings [2,11, 12], and the geometric problem of finding monotone trajectories between two given points in the plane avoiding convex obstacles [3].

Planar monotone drawings with straight-line edges form a natural setting and it is known that biconnected planar graphs and trees always admit such drawings, for some

\footnotetext{
* Research supported in part by the MIUR project AlgoDEEP prot. 2008TFBWL4. Work on these results began at the 6th Bertinoro Workshop on Graph drawing. Discussion with other participants is gratefully acknowledged.
} 
combinatorial embedding of the graph [1]. However, the question whether a simply connected planar graph always admits a planar monotone drawing or not is still open.

On the other hand, in the fixed embedding setting (i.e., the planar embedding of the graph is given as part of the input and the drawing algorithm is not allowed to alter it) it is known [1] that there exist simply connected planar embedded graphs that admit no straight-line monotone drawings.

In this paper we study planar monotone drawings of graphs in the fixed embedding setting, answering the natural question whether monotone drawings with a given constant number of bends per edge can always be computed, and identifying some subclasses of planar graphs that always admit planar monotone drawings with straight-line edges. Our contributions are summarized below:

- We prove that every $n$-vertex planar embedded graph has an embedding-preserving monotone drawing with curve complexity 2 , that is, the maximum number of bends along an edge is 2 , and with at most $4 n-10$ bends in total. Such a drawing can be computed in linear time and has polynomial area.

- We show that our bound on the curve complexity is tight, i.e., two bends per edge are sometimes necessary. More precisely, we describe an infinite family of embedded planar graphs that require two bends on a linear number of edges in any embedding-preserving monotone drawing.

- We investigate what subfamilies of embedded planar graphs can be realized as embedding-preserving monotone drawings with straight-line edges. We prove that biconnected embedded planar graphs and outerplane graphs always admit such a drawing, which can be computed in linear time.

The remainder of the paper is structured as follows. Basic defintions and results are given in Section 2. An algorithm for computing embedding-preserving monotone drawings of general embedded planar graphs with at most two bends per edge is described in Section 3. Algorithms for computing straight-line monotone drawings of meaningful subfamilies of embedded planar graphs are given in Section 4. Concluding remarks and open questions are presented in Section 5. For reasons of space some proofs are sketched or omitted in this extended abstract. Full proofs can be found in the appendix.

\section{Preliminaries}

We assume familiarity with basic concepts of graph drawing (see, e.g., [5]). Let $G$ be a planar graph and let $\phi$ be a planar embedding of $G$. The embedding $\phi$ defines the set of internal faces and the outer face of $G$. For every vertex $v$ of $G$, the embedding $\phi$ also defines the circular clockwise order of the edges incident to $v$. Graph $G$ along with an embedding $\phi$ is called an embedded planar graph, and is denoted by $G_{\phi}$. Any subgraph of $G_{\phi}$ obtained by removing some edges from $G_{\phi}$ is a subgraph that preserves the planar embedding $\phi$. A drawing of $G_{\phi}$ is a planar drawing of $G$ with embedding $\phi$.

A subdivision of a graph $G$ is obtained by replacing each edge of $G$ with a path. A $k$-subdivision of $G$ is such that any path replacing an edge of $G$ has at most $k$ internal vertices. A graph $G$ is connected if every pair of vertices is connected by a path and is biconnected (resp. triconnected) if removing any vertex (resp. any two vertices) leaves 
$G$ connected. In order to handle the decomposition of a biconnected graph into its triconnected components, we use the well-known SPQR-tree data structure [7] (see the appendix for the basic definitions about SPQR-trees).

A monotone drawing $\Gamma$ of a planar graph $G$ (of an embedded planar graph $G_{\phi}$ ) is a drawing of $G$ (of $G_{\phi}$ ) such that for every pair of vertices $u$ and $v$ there exists a path from $u$ to $v$ in $\Gamma$ that is monotone in some direction.

A monotone drawing of any tree $T$ can be constructed in polynomial area by using Algorithm DFS-based [1]. This algorithm relies on the concept of Stern-Brocot tree $[13,4] \mathcal{S B}$, which is an infinite tree whose nodes are in bijective mapping with the irreducible positive rational numbers (basic definitions on the Stern-Brocot tree are recalled in the appendix). Algorithm DFS-based assigns to the edges of the tree $T$ slopes $\frac{1}{1}, \frac{2}{1}, \ldots, \frac{n-1}{1}$ (which are the first $n-1$ elements of the rightmost path of $\mathcal{S B}$ ) according to a DFS-visit of $T$. Polynomial area is ensured by the following property of $\mathcal{S B}$.

Property 1. $[4,13]$ The sum of the numerators of the elements of the $i$-th level of $\mathcal{S B}$ is $3^{i-1}$ and the sum of the denominators of the elements of the $i$-th level of $\mathcal{S B}$ is $3^{i-1}$.

The following property is also satisfied by any monotone drawing $\Gamma$ of a tree $T$.

Property 2. [1] Any drawing $\Gamma^{\prime}$ of $T$ such that the slopes of each edge $e \in T$ in $\Gamma^{\prime}$ is the same as the slope of $e$ in $\Gamma$ is monotone. Also, the slopes of any two leaf-edges $e^{\prime}$ and $e^{\prime \prime}$ of $T$ in $\Gamma$ are such that $e^{\prime}$ and $e^{\prime \prime}$ diverge, that is, the elongations of $e^{\prime}$ and $e^{\prime \prime}$ do not cross each other.

The turn angle from the edge $(u, v)$ to the edge $(v, w)$ is the smallest angle between the half-line from $u$ through $v$ and the edge $(v, w)$; see Fig. 1. The turn angle is positive if the rotation defined by this angle is clockwise and negative if it is counterclockwise.
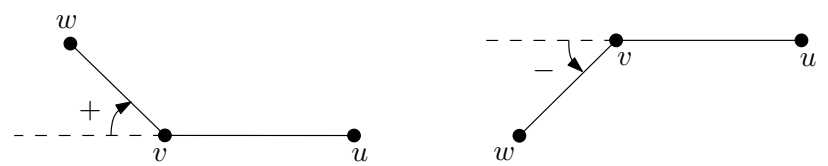

Fig. 1: A positive and a negative turn angles from edge $(u, v)$ to edge $(v, w)$.

\section{Monotone Drawings with Bends of Embedded Planar Graphs}

In this section we study monotone drawings of embedded planar graphs. We remark that it is still unknown whether every planar graph admits a straight-line monotone drawing in the variable embedding setting, while it is known that straight-line monotone drawings do not always exist if the embedding of the graph is fixed [1]. We therefore investigate monotone drawings with bends along some edges, and we show that two bends per edge are always sufficient and sometimes necessary for the existence of a monotone drawing in the fixed embedding setting. 
We need some preliminary definitions. An upright spanning tree $T$ of an embedded planar graph $G_{\phi}$ is a rooted ordered spanning tree of $G_{\phi}$ such that: $(i) T$ preserves the planar embedding of $G_{\phi} ;(i i)$ the root of $T$ is a vertex $r$ of the outer face of $G_{\phi} ;(i i i)$ there exists a planar drawing of $G_{\phi}$ that contains an upward drawing of $T$ such that no edge goes below $r$. Fig. 2(b) and (c) show two different ordered spanning trees of the embedded planar graph of Fig. 2(a): The first one is an upright spanning tree, while the second is not. Given an embedded planar graph $G_{\phi}$, an upright spanning tree $T$ of $G_{\phi}$ can be computed as follows. Construct any planar straight-line drawing $\Gamma$ of $G_{\phi}$. Orient the edges of $G_{\phi}$ in $\Gamma$ according to the upward direction. Let $r$ be a vertex on the outer face of $G_{\phi}$ with the smallest $y$-coordinate in $\Gamma$. Then, compute any spanning tree $T$ of $G_{\phi}$ rooted at $r$ such that the left-to-right order of the children of $r$ in $T$ is consistent with the left-to-right order of the neighbors of $r$ in $\Gamma$ and the left-to-right order of the children of each vertex $w$ in $T$ is consistent with the clockwise order of the neighbors of $w$ in $G_{\phi}$, computed starting from the edge connecting $w$ to its parent in $T$.

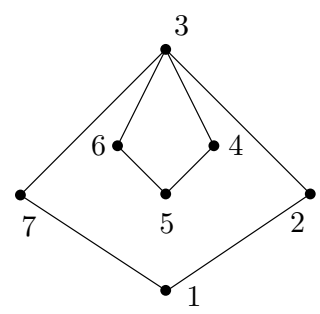

(a)

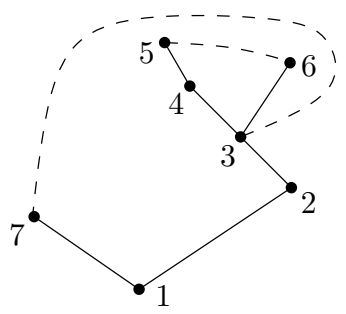

(b)

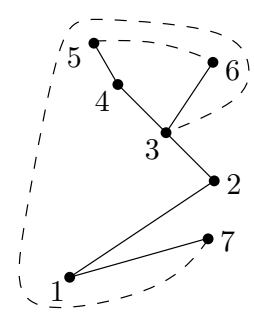

(c)

Fig. 2: (a) A drawing $\Gamma$ of an embedded planar graph $G_{\phi}$. (b) An upright spanning tree of $G_{\phi}$. (c) A spanning tree of $G_{\phi}$ that is not upright.

Let $T$ be an upright spanning tree of $G_{\phi}$. The rgbb-coloring of $G_{\phi}$ with respect to $T$ is a coloring of the edges of $G_{\phi}$ with four colors (red, green, blue, and black) such that: An edge is colored black if it belongs to $T$; an edge is colored green if it connects two leaves of $T$; an edge is colored red if it connects a leaf to an internal vertex of $T$; an edge is colored blue if it connects two internal vertices of $T$.

We denote by $C\left(G_{\phi}, T\right)$ the rgbb-coloring of $G_{\phi}$ with respect to $T$. We prove the following lemma.

Lemma 1. Let $G_{\phi}$ be an embedded planar graph with $n$ vertices, let $T$ be an upright spanning tree of $G_{\phi}$, and let $C\left(G_{\phi}, T\right)$ be the rgbb-coloring of $G_{\phi}$ with respect to $T$. Then we can compute a monotone drawing $\Gamma$ of $G_{\phi}$ such that each black or green edge of $C\left(G_{\phi}, T\right)$ is drawn as a straight-line segment, each red edge has 1 bend, and each blue edge has 2 bends. The running time of the algorithm is $O(n)$ and the drawing $\Gamma$ has $O(n) \times O\left(n^{2}\right)$ area.

Proof. First, starting from $G_{\phi}$ and $T$, construct a graph $G_{\phi}^{\prime}$ and an upright spanning tree $T^{\prime}$ of $G_{\phi}^{\prime}$ such that: (i) $G_{\phi}^{\prime}$ is a 2 -subdivision of $G_{\phi}$, (ii) $T$ is a subtree of $T^{\prime}$, and 
(iii) all the edges of $G_{\phi}^{\prime}$ that are not in $T^{\prime}$ connect two leaves of $T^{\prime}$. Fig. 3(a) and (b) show a graph $G_{\phi}$ with an upright spanning tree $T$ and the corresponding graph $G_{\phi}^{\prime}$ with its upright spanning tree $T^{\prime}$ satisfying (i)-(iii). Then, the monotone drawing of $G_{\phi}$ with curve complexity 2 is constructed by first computing a straight-line monotone drawing of $G_{\phi}^{\prime}$ and then replacing each subdivision vertex with a bend; see Fig. 3(c).

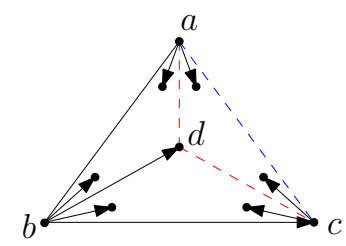

(a)

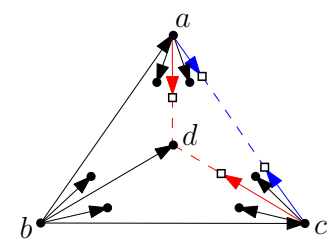

(b)

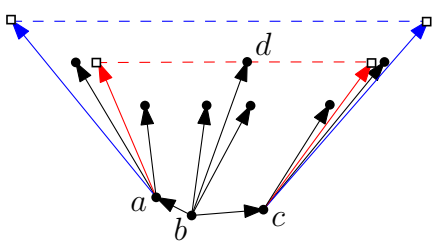

(c)

Fig. 3: (a) A graph $G_{\phi}$ with an upright spanning tree $T$ rooted at vertex $b$. Solid edges belong to $T$, while dashed edges do not. (b) The corresponding graph $G_{\phi}^{\prime}$ with its upright spanning tree $T^{\prime}$. Solid edges belong to $T^{\prime}$, while dashed edges do not. Subdivision vertices are drawn as squares. (c) A straight-line monotone drawing of $G_{\phi}^{\prime}$ that corresponds to a monotone drawing of $G_{\phi}$ with bent edges.

Graphs $G_{\phi}^{\prime}$ and $T^{\prime}$ are constructed as follows. Initialize $G_{\phi}^{\prime}=G_{\phi}$ and $T^{\prime}=T$. Subdivide each red edge $(s, t)$ of $G_{\phi}^{\prime}$ with a vertex $k$ and add edge $(t, k)$ to $T^{\prime}$, where $t$ is the internal vertex of $T^{\prime}$. Subdivide each blue edge $(s, t)$ of $G_{\phi}^{\prime}$ twice, with two vertices $k$ and $z$, and add edges $(s, k)$ and $(t, z)$ to $T^{\prime}$.

The straight-line monotone drawing of $G_{\phi}^{\prime}$ is computed in two steps. First, with Algorithm DFS-based [1], we construct a straight-line monotone drawing of $T^{\prime}$, and then we add the remaining (non-tree) edges as straight-line segments, which results in using two segments for red edges and three segments for blue edges.

To argue the monotonicity for non-tree edges, recall that, by Property 2 , it is possible to elongate the edges of $T^{\prime}$ without affecting monotonicity and planarity.

Further, as Algorithm DFS-based assigns slopes $\frac{1}{1}, \frac{2}{1}, \ldots, \frac{n-1}{1}$ to the edges of $T^{\prime}$, the elongation of each leaf-edge $(u, v)$ intersects each vertical line $x=k$, where $k$ is any integer value greater than the $x$-coordinate of $u$, at an integer grid point. Moreover, as by Property 2 the leaf-edge elongations diverge, such intersections appear in the same order on each vertical line $x=k^{\prime}$, where $k^{\prime}$ is any integer value greater than the $x$-coordinate of every internal vertex of $T^{\prime}$; see Fig. 4(a).

Another key observation is that the graph $G_{L}$ induced by the leaves of $T^{\prime}$ is outerplanar and can be augmented, by adding dummy edges, to a biconnected outerplanar graph in which each internal face is a 3-cycle in such a way that the order of the vertices on the outer face is the same as the left-to-right order of the leaves of $T^{\prime}$; see Fig. 4(b).

The vertices of $G_{L}$ are assigned levels in such a way that each edge of $G_{L}$ connects two vertices that are either on the same level or on adjacent levels. Let $l$ be the number of levels of $G_{L}$. Then, place all the vertices at level $i$, with $i=1, \ldots, l$, on a vertical line $x=k+l-i+1$, where $k$ is the $x$-coordinate of the rightmost internal vertex of $T^{\prime}$. 
Note that this placement, together with the fact that each such vertical line intersects the elongations of all the leaf-edges in the same order, ensures the planarity of the straightline drawing of $G_{L}$. Further, as the order of the vertices on the outer face of $G_{L}$ is the same as the left-to-right order of the leaves of $T^{\prime}$, the edges of $T^{\prime}$ do not cross any edge of $G_{L}$, hence ensuring the planarity of $G_{\phi}^{\prime}$; see Fig. 4(c).

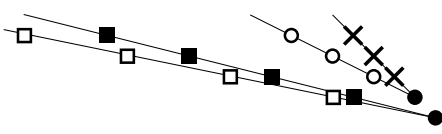

(a)

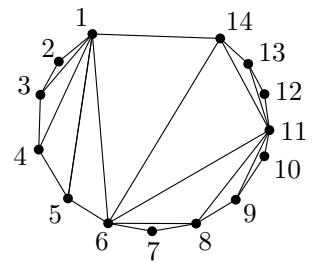

(b)

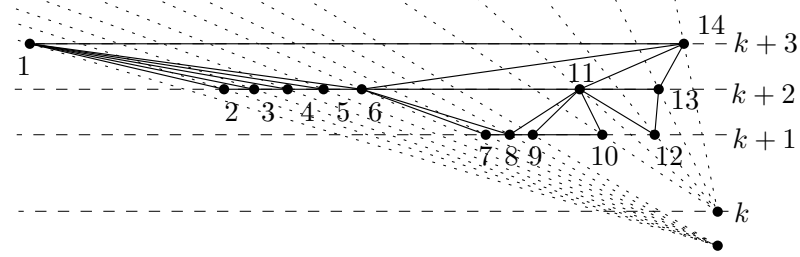

(c)

Fig. 4: For space and readability, the drawings in (a) and (c) are rotated to $90^{\circ}$ and the grid unit distances in (c) are not uniform. (a) The elongations of the leaf-edges have integer intersections with all the vertical lines in the same order. (b) An augmented graph $G_{L}$. (c) The drawing of $G_{L}$ (where $l=3$ ).

The assignment of levels is performed as follows. Assign level 1 to the first and to the last vertex in the left-to-right order of the leaves of $T^{\prime}$. Note that, these two vertices are connected by an edge, as $G_{L}$ is a biconnected outerplanar graph and the order of the vertices on its outer face is the same as the left-to-right order of the leaves of $T^{\prime}$. Then, starting from such an edge, consider any edge $(u, v)$ on the outer face of the graph induced by the vertices whose level has been already assigned and consider the unique vertex $w$ that is connected to both $u$ and $v$, and whose level has not been assigned yet, if any. Note that, either $u$ and $v$ have the same level $i$ or one of them has level $i$ and the other has level $i+1$. In both cases, assign level $i+1$ to $w$, as shown in Fig. 4(b) and (c).

The drawing of $G_{\phi}^{\prime}$ is monotone because between any two vertices there exists a monotone path composed only of edges of $T^{\prime}$, while edges not in $T^{\prime}$ do not affect the monotonicity. Hence, monotonicity is maintained when dummy edges are removed. Note that, any monotone path traversing a leaf-edge of $T^{\prime}$ has the corresponding leaf as an end-vertex. If the leaf is a subdivision vertex of any non-black edge, it does not belong to $G_{\phi}$. Hence, all the monotone paths in $G_{\phi}$ are composed only of edges of $T$, 
whose drawing is monotone since it is a subtree of $T^{\prime}$. Therefore, the drawing of $G_{\phi}$ is monotone, each red edge has one bend, and each blue edge has two bends.

In order to compute the area of the obtained drawing, recall that Algorithm DFSbased [1] produces a drawing of $T^{\prime}$ in $O(n) \times O\left(n^{2}\right)$ area. Since the number of vertical lines added to host the drawing of $G_{L}$ is equal to the number $l$ of levels assigned to the vertices of $G_{L}$, and since $l$ is bounded by the number of leaves, which is $O(n)$, the area of the whole drawing is still $O(n) \times O\left(n^{2}\right)$.

It is easy to see that the drawing can be computed in $O(n)$ time, by considering the individual steps. The computation of the three necessary graphs, $T, G_{\phi}^{\prime}$ and $T^{\prime}$, can be performed in linear time. Also, the slopes of the edges of $T^{\prime}$ can be computed in linear time with Algorithm DFS-based [1] by constructing the Stern-Brocot tree and by performing a rightmost DFS visit of it. Further, graph $G_{L}$ can be augmented in linear time. Finally, the assignment of levels to the vertices of $G_{L}$ is also performed in linear time, as each vertex is considered just once and its level is assigned only based on the levels of its two neighbors. This runtime analysis concludes the proof of Lemma 1 .

Note that, according to Lemma 1 there always exists a monotone drawing $\Gamma$ of $G_{\phi}$ with curve complexity 2 and at most $4 n-10$ bends in total. Indeed, $G_{\phi}$ has at most $3 n-6$ edges and every spanning tree of $G_{\phi}$ has $n-1$ edges. Then, using the algorithm described in the proof of Lemma $1, \Gamma$ has at most $2(3 n-6-n+1)=4 n-10$ edges in total. Moreover, this upper bound is asymptotically tight, as there exist embedded planar graphs that require a linear total number of bends in any monotone drawing. Namely, we first prove in Lemma 2 that there exist embedded planar graphs requiring at least one bend on some edges. Then, based on this lemma, we prove in Lemma 3 that there exist infinitely many embedded planar graphs whose monotone drawings require two bends on a linear number of edges.

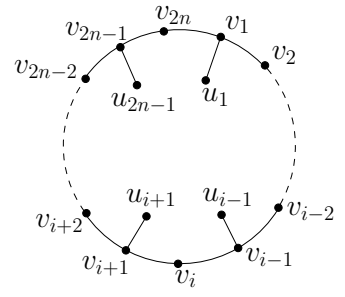

(a)

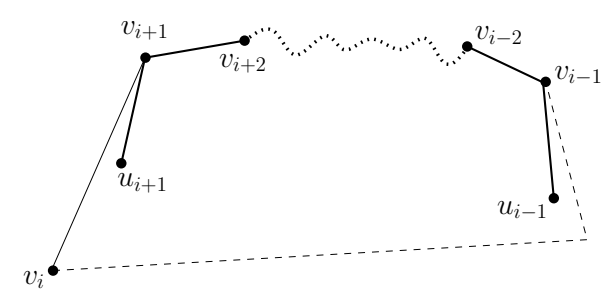

(b)

Fig. 5: (a) A graph $G_{\phi}$ with $3 n$ vertices that does not admit any straight-line monotone drawing. (b) Edges $\left(v_{i-1}, v_{i}\right)$ and $\left(v_{i}, v_{i+1}\right)$ can not be drawn as straight-line segments.

Lemma 2. For every $n \geq 3$ there exists an embedded planar graph $G_{\phi}$ with $3 n$ vertices and $3 n$ edges that does not admit any straight-line monotone drawing.

Sketch of Proof: We describe an embedded planar graph $G_{\phi}$ that does not admit any straight-line monotone drawing (refer to Fig. 5(a)). $G_{\phi}$ consists of a simple cycle $C=$ 
$v_{1}, \ldots, v_{2 n}$ of length $2 n$ and of $n$ vertices $u_{1}, u_{3}, \ldots, u_{2 n-1}$ of degree 1 , called legs, incident to the vertices $v_{1}, v_{3}, \ldots, v_{2 n-1}$ of $C$ with odd indices, respectively. The embedding of $G_{\phi}$ is such that all the legs are inside $C$, that is, they are inside the unique internal face of $C$. As any two consecutive legs $\left(v_{i-1}, u_{i-1}\right)$ and $\left(v_{i+1}, u_{i+1}\right)$ diverge, it is not possible to connect vertices $v_{i-1}$ and $v_{i+1}$ by drawing edges $\left(v_{i-1}, v_{i}\right)$ and $\left(v_{i}, v_{i+1}\right)$ as straight-line segments. Refer to Fig. 5(b).

The next lemma shows that there are infinitely many embedded planar graphs that require two bends per edge on a linear number of edges in any embedding-preserving monotone drawing.

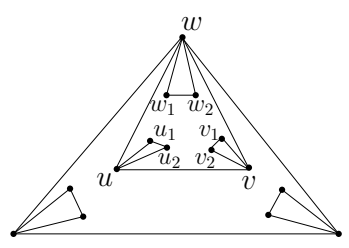

(a)

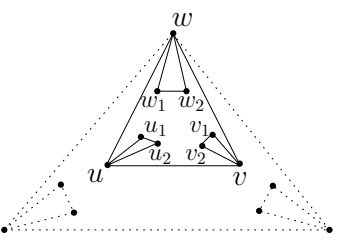

(b)

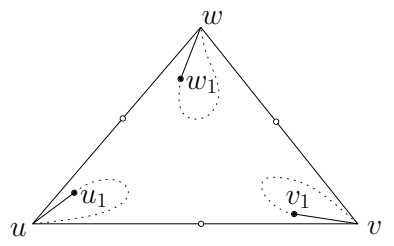

(c)

Fig. 6: (a) An example of a graph $G_{\phi}$ with $n=15$ vertices, that coincides with a graph $G_{\phi}^{3}$ constructed from $G_{\phi}^{2}$ by adding vertices $u_{1}, u_{2}, v_{1}, v_{2}, w_{1}, w_{2}$ inside triangular face $u, v, w$. (b) A subgraph $G_{\phi}^{t}$ of $G_{\phi}$ induced by a triangle $(u, v, w)$ and all the vertices inside it. (c) A subdivision (white circles) of the subgraph $G_{\phi}^{h}$ (solid edges) of $G_{\phi}^{t}$ induced by $u, v, w, u_{1}, v_{1}, w_{1}$. By Lemma 2, this subdivision does not admit any straight-line monotone drawing.

Lemma 3. For every odd $n \geq 9$ there exists an embedded planar graph $G_{\phi}$ with $n$ vertices and $\frac{3}{2}(n-1)$ edges such that every monotone drawing of $G_{\phi}$ has at least $\frac{n-3}{6}$ edges with at least two bends and thus at least $\frac{n-3}{3}$ bends in total.

Sketch of Proof: Refer to Fig. 6. Consider an odd integer $n \geq 9$. We construct $G_{\phi}$ iteratively. Let $G_{\phi}^{1}$ be a triangle graph. Graph $G_{\phi}^{i}$ is constructed from $G_{\phi}^{i-1}$ as follows. Initialize $G_{\phi}^{i}=G_{\phi}^{i-1}$. Let $(u, v, w)$ be a triangular internal face of $G_{\phi}^{i}$. Add 6 new vertices $u_{1}, u_{2}, v_{1}, v_{2}, w_{1}, w_{2}$ and 9 new edges $\left(u, u_{1}\right),\left(u, u_{2}\right),\left(u_{1}, u_{2}\right),\left(v, v_{1}\right),\left(v, v_{2}\right)$, $\left(v_{1}, v_{2}\right),\left(w, w_{1}\right),\left(w, w_{2}\right),\left(w_{1}, w_{2}\right)$ to $G_{\phi}^{i}$ in such a way that all the new vertices are inside $(u, v, w)$. Note that the $n$-vertex graph $G_{\phi}^{i}$ is planar and has $\frac{3}{2}(n-1)$ edges. Any monotone drawing of $G_{\phi}$ has at least $\frac{n-3}{6}$ edges with at least two bends.

Lemma 1 and Lemma 3 together provide a tight bound on the curve complexity of monotone drawings in the fixed embedding setting. The next theorem summarizes the main contribution of this section.

Theorem 1. Every embedded planar graph with $n$ vertices admits a monotone drawing with curve complexity 2 , at most $4 n-10$ bends in total, and $O(n) \times O\left(n^{2}\right)$ area; such a drawing can be computed in $O(n)$ time. Also, there exist infinitely many embedded planar graphs any monotone drawing of which requires two bends on $\Omega(n)$ edges. 


\section{Monotone Drawings with Straight-line Edges}

In this section we prove that there exist meaningful subfamilies of embedded planar graphs that can be realized as straight-line monotone drawings. In particular, we prove that both the class of outerplane graphs and the class of embedded planar biconnected graphs have this property.

\subsection{Outerplane Graphs}

An embedded planar graph $G_{\phi}$ is an outerplane graph if all its vertices are on the outer face. We prove the following result.

Theorem 2. Every outerplane graph admits a straight-line monotone drawing. Also, there exists an algorithm that computes such a drawing in $O(n)$ time and $O(n) \times O\left(n^{2}\right)$ area.

Proof. Let $T$ be an upright spanning tree of $G_{\phi}$ obtained by performing a "rightmost DFS" visit of $G_{\phi}$; see Fig. 7(a). Consider a decomposition of $G_{\phi}$ into its maximal biconnected components. Observe that, for each maximal biconnected component $B$ that is connected to the root of $T$ through a cut-vertex $v, T$ contains all the edges of $B$ except for the internal chords (dashed edges in Fig. 7(a)) and for the leftmost edge incident to $v$ (dotted edges in Fig. 7(a)).

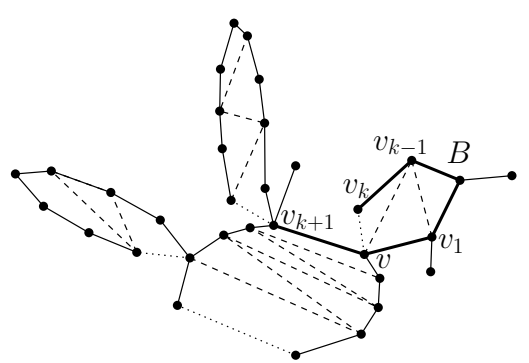

(a)

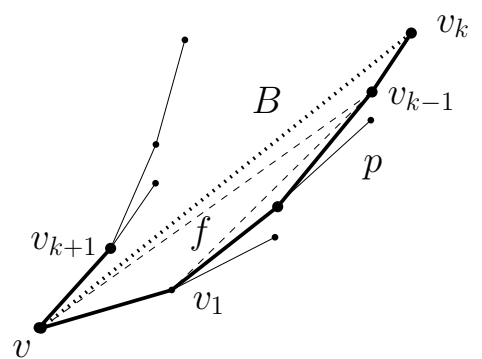

(b)

Fig. 7: (a) An outerplane graph $G_{\phi}$ and the upright spanning tree $T$ of $G_{\phi}$ obtained by performing a "rightmost DFS" visit. Edges of $T$ are represented as solid segments. (b) A strictly convex drawing of a maximal biconnected component $B$ of $G_{\phi}$.

A straight-line monotone drawing of $G_{\phi}$ is constructed by first computing a straightline monotone drawing of $T$, with Algorithm DFS-based [1], and then reinserting the edges not in $T$ as straight-line segments. In order to reinsert such edges, for each maximal biconnected component $B$, consider the path $p=\left(v, v_{1}, \ldots, v_{k}\right)$ that is composed of the edges belonging both to $B$ and to $T$.

According to Algorithm DFS-based [1] the slopes of the edges of $p$ are all positive and increasing with respect to the distance from $v$ in $p$. Hence, path $p$ is drawn in $T$ 
as a polygonal line that is convex on the left side, that is, the straight-line segment connecting any two non-consecutive vertices of $p$ completely lies to the left of $p$; see Fig. 7(b). Thus, reinserting edge $\left(v, v_{k}\right)$ as the straight-line segment between $v$ and $v_{k}$ determines that $\left(v, v_{k}\right)$ is the leftmost edge of $B$ incident to $v$ in the drawing and that the boundary of $B$, that is, the cycle composed of the edges of $p$ plus $\left(v, v_{k}\right)$, delimits a strictly-convex region $f$.

We show that $f$ does not contain any other vertex of $T$. Namely, the vertex $v_{k+1}$ such that edge $\left(v, v_{k+1}\right)$ follows $\left(v, v_{1}\right)$ in the counter-clockwise order of the edges around $v$ in $T$ lies outside $f$. This is due to the fact that, according to Algorithm DFSbased, the slope of $\left(v, v_{k+1}\right)$ is greater than the slope of $\left(v_{k-1}, v_{k}\right)$ which, in its turn, is greater than the slope of $\left(v, v_{k}\right)$; see Fig. 7(b).

Hence, $f$ is an empty strictly-convex region, and the chords of $B$ can be reinserted as straight-line segments while maintaining planarity.

The area of the drawing is the same as the area of $T$ computed by Algorithm DFSbased, namely $O(n) \times O\left(n^{2}\right)$. The drawing can be computed in $O(n)$ time. Namely, drawing $T$ by using Algorithm DFS-based takes $O(n)$ time [1], and the same holds for reinserting missing edges.

\subsection{Biconnected Graphs}

It is known [1] that straight-line monotone drawings of biconnected planar graphs in the variable embedding setting can always be computed. This result is obtained by means of an algorithm that exploits SPQR-trees and that preserves any given embedding, as long as the graph contains no parallel component whose poles are connected by an edge. However, this algorithm can be easily modified in order to compute monotone drawings with curve complexity 1 of every embedded biconnected planar graph, as the edges connecting the poles of a parallel component could be placed in their correct position by adding a bend, when necessary.

In this section we prove that in fact we can compute a monotone drawing of every embedded biconnected planar graph with no bends at all.

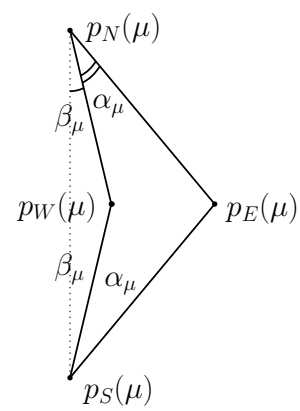

(a)

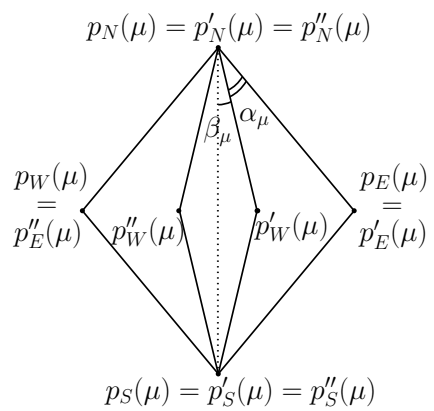

(b)

Fig. 8: (a) A boomerang. (b) A diamond. 
As for the variable-embedding setting case [1], our algorithm relies on a bottom-up visit of the SPQR-tree of the biconnected graph $G$ in which at each step a drawing of the pertinent graph of the currently considered node $\mu$ is constructed inside a boomerang $\operatorname{boom}(\mu)$, that is, a quadrilateral composed of points $p_{N}(\mu), p_{E}(\mu), p_{S}(\mu)$, and $p_{W}(\mu)$ such that $p_{W}(\mu)$ is inside triangle $\triangle\left(p_{N}(\mu), p_{S}(\mu), p_{E}(\mu)\right)$ and $2 \alpha_{\mu}+\beta_{\mu}<\frac{\pi}{2}$, where $\alpha_{\mu}=p_{W}(\mu) \widehat{p_{S}(\mu)} p_{E}(\mu)=p_{W}(\mu) \widehat{p_{N}(\mu)} p_{E}(\mu)$ and $\beta_{\mu}=p_{W}(\mu) \widehat{p_{S}(\mu)} p_{N}(\mu)=$ $p_{W}(\mu) \widehat{p_{N}(\mu)} p_{S}(\mu)$; see Fig. 8(a).

In order to cope with the fixed-embedding setting, we introduce a new shape, called diamond and denoted by $\operatorname{diam}(\mu)$, that is a convex quadrilateral $\left(p_{N}(\mu), p_{E}(\mu), p_{S}(\mu)\right.$, $\left.p_{W}(\mu)\right)$ composed of two boomerangs boom ${ }^{\prime}(\mu)=\left(p_{N}^{\prime}(\mu), p_{E}^{\prime}(\mu), p_{S}^{\prime}(\mu), p_{W}^{\prime}(\mu)\right)$ and boom ${ }^{\prime \prime}(\mu)=\left(p_{N}^{\prime \prime}(\mu), p_{E}^{\prime \prime}(\mu), p_{S}^{\prime \prime}(\mu), p_{W}^{\prime \prime}(\mu)\right)$ such that $p_{N}(\mu)=p_{N}^{\prime}(\mu)=p_{N}^{\prime \prime}(\mu)$, $p_{S}(\mu)=p_{S}^{\prime}(\mu)=p_{S}^{\prime \prime}(\mu), p_{E}(\mu)=p_{E}^{\prime}(\mu)$ and $p_{W}(\mu)=p_{E}^{\prime \prime}(\mu)$; see Fig. 8(b). We have the following.

Theorem 3. Every biconnected embedded planar graph admits a straight-line monotone drawing, which can be computed in linear time.

\section{Conclusions and Open Problems}

In this paper we studied monotone drawings of graphs in the fixed embedding setting. Since not all embedded planar graphs admit an embedding-preserving monotone drawing with straight-line edges, we focused on computing embedding-preserving monotone drawings with low curve complexity. We proved that curve complexity 2 always suffices and that this bound is worst-case optimal. Furthermore, we described algorithms for computing straight-line monotone drawings for meaningful subfamilies of embedded planar graphs. All the algorithms presented in this paper can be performed in linear time and most of them produce drawings which require polynomial area.

The results in this paper naturally give rise to several interesting open problems; some of them are listed below.

\section{Existential Questions}

Problem 1. Finding meaningful subfamilies of embedded planar graphs (other than outerplane graphs and embedded biconnected graphs) that admit monotone drawings with curve complexity smaller than 2 .

Problem 2. Is it possible to characterize the embedded planar graphs that admit monotone drawings with curve complexity smaller than 2 ?

\section{Complexity Questions}

Problem 3. Given an embedded planar graph $G_{\phi}$ and an integer $k \in\{0,1\}$, what is the complexity of deciding whether $G_{\phi}$ admits a monotone drawing with curve complexity $k$ ? 
Problem 4. Given a graph $G$ and an integer $k \in\{0,1\}$, what is the complexity of deciding whether there exists an embedding $\phi$ such that $G_{\phi}$ admits a monotone drawing with curve complexity $k$ ?

Problem 5. Given a graph $G$ and an integer $k \in\{0,1\}$, what is the complexity of deciding whether there exists an embedding $\phi$ such that $G_{\phi}$ does not admit any monotone drawing with curve complexity $k$ ?

Notice that, although Problems 3-5 are related, there is no evidence that answering one of them implies an answer for any other.

\section{Algorithmic Questions}

Problem 6. Is there any algorithm that computes monotone drawings of embedded biconnected planar graphs in polynomial area?

Problem 7. Is there any algorithm that computes monotone drawings of outerplane graphs in subcubic area?

\section{References}

1. P. Angelini, E. Colasante, G. D. Battista, F. Frati, and M. Patrignani. Monotone drawings of graphs. In U. Brandes and S. Cornelsen, editors, Graph Drawing (GD'10), volume 6502, pages 13-24, 2011.

2. P. Angelini, F. Frati, and L. Grilli. An algorithm to construct greedy drawings of triangulations. J. Graph Algorithms Appl., 14(1):19-51, 2010.

3. E. M. Arkin, R. Connelly, and J. S. B. Mitchell. On monotone paths among obstacles with applications to planning assemblies. In Symposium on Computational Geometry, pages 334 343, 1989.

4. A. Brocot. Calcul des rouages par approximation, nouvelle methode. Revue Chronometrique, 6:186-194, 1860.

5. G. Di Battista, P. Eades, R. Tamassia, and I. G. Tollis. Graph Drawing. Prentice Hall, Upper Saddle River, NJ, 1999.

6. G. Di Battista and R. Tamassia. On-line maintenance of triconnected components with SPQR-trees. Algorithmica, 15(4):302-318, 1996.

7. G. Di Battista and R. Tamassia. On-line planarity testing. SIAM J. Comput., 25:956-997, 1996.

8. A. Garg and R. Tamassia. Upward planarity testing. Order, 12:109-133, 1995.

9. C. Gutwenger and P. Mutzel. A linear time implementation of SPQR-trees. In J. Marks, editor, Graph Drawing (GD '00), volume 1984 of $L N C S$, pages 77-90, 2001.

10. W. Huang, P. Eades, and S.-H. Hong. A graph reading behavior: Geodesic-path tendency. In PacificVis, pages 137-144, 2009.

11. T. Leighton and A. Moitra. Some results on greedy embeddings in metric spaces. Discrete \& Computational Geometry, 44(3):686-705, 2010.

12. C. H. Papadimitriou and D. Ratajczak. On a conjecture related to geometric routing. Theor. Comput. Sci., 344(1):3-14, 2005.

13. M. A. Stern. Ueber eine zahlentheoretische funktion. Journal fur die reine und angewandte Mathematik, 55:193-220, 1858. 


\section{Appendix A: SPQR-Trees and the Stern-Brocot Tree}

SPQR-trees have been introduce by Di Battista and Tamassia (see, e.g., [6, 7]). Here we recall some basic definitions about SPQR-trees. Further details can be found in $[6,7,9]$.

A graph is st-biconnectible if adding edge $(s, t)$ to it yields a biconnected graph. Let $G$ be an st-biconnectible graph. A separation pair of $G$ is a pair of vertices whose removal disconnects the graph. A split pair of $G$ is either a separation pair or a pair of adjacent vertices. A maximal split component of $G$ with respect to a split pair $\{u, v\}$ (or, simply, a maximal split component of $\{u, v\})$ is either an edge $(u, v)$ or a maximal subgraph $G^{\prime}$ of $G$ such that $G^{\prime}$ contains $u$ and $v$, and $\{u, v\}$ is not a split pair of $G^{\prime}$. A vertex $w \neq u, v$ belongs to exactly one maximal split component of $\{u, v\}$. We call split component of $\{u, v\}$ the union of any number of maximal split components of $\{u, v\}$.

In the paper, we assume that any SPQR-tree of a graph $G$ is rooted at one edge of $G$, called the reference edge.

The rooted SPQR-tree $\mathcal{T}$ of a biconnected graph $G$, with respect to a reference edge $e$, describes a recursive decomposition of $G$ induced by its split pairs. The nodes of $\mathcal{T}$ are of four types: $\mathrm{S}, \mathrm{P}, \mathrm{Q}$, and $\mathrm{R}$. Their connections are called $\operatorname{arcs}$, in order to distinguish them from the edges of $G$.

Each node $\mu$ of $\mathcal{T}$ has an associated st-biconnectible multigraph, called the skeleton of $\mu$ and denoted by skeleton $(\mu)$. Skeleton skeleton $(\mu)$ shows how the children of $\mu$, represented by "virtual edges", are arranged into $\mu$. The virtual edge in skeleton $(\mu)$ associated with a child node $\nu$, is called the virtual edge of $\nu$ in skeleton $(\mu)$.

For each virtual edge $e_{i}$ of skeleton $(\mu)$, recursively replace $e_{i}$ with the skeleton skeleton $\left(\mu_{i}\right)$ of its corresponding child $\mu_{i}$. The subgraph of $G$ that is obtained in this way is the pertinent graph of $\mu$ and is denoted by pertinent $(\mu)$.

Given a biconnected graph $G$ and a reference edge $e=\left(u^{\prime}, v^{\prime}\right)$, tree $\mathcal{T}$ is recursively defined as follows. At each step, a split component $G^{*}$, a pair of vertices $\{u, v\}$, and a node $\nu$ in $\mathcal{T}$ are given. A node $\mu$ corresponding to $G^{*}$ is introduced in $\mathcal{T}$ and attached to its parent $\nu$. Vertices $u$ and $v$ are the poles of $\mu$ and denoted by $u(\mu)$ and $v(\mu)$, respectively. The decomposition possibly recurs on some split components of $G^{*}$. At the beginning of the decomposition $G^{*}=G-\{e\},\{u, v\}=\left\{u^{\prime}, v^{\prime}\right\}$, and $\nu$ is a Q-node corresponding to $e$.

Base Case: If $G^{*}$ consists of exactly one edge between $u$ and $v$, then $\mu$ is a Q-node whose skeleton is $G^{*}$ itself.

Parallel Case: If $G^{*}$ is composed of at least two maximal split components $G_{1}, \ldots, G_{k}$ $(k \geq 2)$ of $G$ with respect to $\{u, v\}$, then $\mu$ is a P-node. Graph skeleton $(\mu)$ consists of $k$ parallel virtual edges between $u$ and $v$, denoted by $e_{1}, \ldots, e_{k}$ and corresponding to $G_{1}, \ldots, G_{k}$, respectively. The decomposition recurs on $G_{1}, \ldots, G_{k}$, with $\{u, v\}$ as pair of vertices for every graph, and with $\mu$ as parent node.

Series Case: If $G^{*}$ is composed of exactly one maximal split component of $G$ with respect to $\{u, v\}$ and if $G^{*}$ has cutvertices $c_{1}, \ldots, c_{k-1}(k \geq 2)$, appearing in this order on a path from $u$ to $v$, then $\mu$ is an S-node. Graph skeleton $(\mu)$ is the path $e_{1}, \ldots, e_{k}$, where virtual edge $e_{i}$ connects $c_{i-1}$ with $c_{i}(i=2, \ldots, k-1)$, $e_{1}$ connects $u$ with $c_{1}$, and $e_{k}$ connects $c_{k-1}$ with $v$. The decomposition recurs on 
the split components corresponding to each of $e_{1}, e_{2}, \ldots, e_{k-1}, e_{k}$ with $\mu$ as parent node, and with $\left\{u, c_{1}\right\},\left\{c_{1}, c_{2}\right\}, \ldots,\left\{c_{k-2}, c_{k-1}\right\},\left\{c_{k-1}, v\right\}$ as pair of vertices, respectively.

Rigid Case: If none of the above cases applies, the purpose of the decomposition step is that of partitioning $G^{*}$ into the minimum number of split components and recurring on each of them. We need some further definition. Given a maximal split component $G^{\prime}$ of a split pair $\{s, t\}$ of $G^{*}$, a vertex $w \in G^{\prime}$ properly belongs to $G^{\prime}$ if $w \neq s, t$. Given a split pair $\{s, t\}$ of $G^{*}$, a maximal split component $G^{\prime}$ of $\{s, t\}$ is internal if neither $u$ nor $v$ (the poles of $G^{*}$ ) properly belongs to $G^{\prime}, e x$ ternal otherwise. A maximal split pair $\{s, t\}$ of $G^{*}$ is a split pair of $G^{*}$ that is not contained into an internal maximal split component of any other split pair $\left\{s^{\prime}, t^{\prime}\right\}$ of $G^{*}$. Let $\left\{u_{1}, v_{1}\right\}, \ldots,\left\{u_{k}, v_{k}\right\}$ be the maximal split pairs of $G^{*}(k \geq 1)$ and, for $i=1, \ldots, k$, let $G_{i}$ be the union of all the internal maximal split components of $\left\{u_{i}, v_{i}\right\}$. Observe that each vertex of $G^{*}$ either properly belongs to exactly one $G_{i}$ or belongs to some maximal split pair $\left\{u_{i}, v_{i}\right\}$. Node $\mu$ is an R-node. Graph skeleton $(\mu)$ is the graph obtained from $G^{*}$ by replacing each subgraph $G_{i}$ with the virtual edge $e_{i}$ between $u_{i}$ and $v_{i}$. The decomposition recurs on each $G_{i}$ with $\mu$ as parent node and with $\left\{u_{i}, v_{i}\right\}$ as pair of vertices.

For each node $\mu$ of $\mathcal{T}$, the construction of skeleton $(\mu)$ is completed by adding a virtual edge $(u, v)$ representing the rest of the graph.

The SPQR-tree $\mathcal{T}$ of a graph $G$ with $n$ vertices and $m$ edges has $m$ Q-nodes and $O(n) \mathrm{S}-, \mathrm{P}-$, and R-nodes. Also, the total number of vertices of the skeletons stored at the nodes of $\mathcal{T}$ is $O(n)$. Finally, SPQR-trees can be constructed and handled efficiently. Namely, given a biconnected planar graph $G$, the SPQR-tree $\mathcal{T}$ of $G$ can be computed in linear time $[6,7,9]$.

The Stern-Brocot tree $[13,4] \mathcal{S B}$ is an infinite tree whose nodes are in bijective mapping with the irreducible positive rational numbers. Tree $\mathcal{S B}$ has two nodes $\frac{0}{1}$ and $\frac{1}{0}$, connected to the same node $\frac{1}{1}$ that is the right child of $\frac{0}{1}$ and the left child of $\frac{1}{0}$. An ordered binary tree is then rooted at $\frac{1}{1}$ as follows. Consider a node $\frac{y}{x}$ of the tree. The left child of $\frac{y}{x}$ is the node $\frac{\left(y+y^{\prime}\right)}{\left(x+x^{\prime}\right)}$, where $\frac{y^{\prime}}{x^{\prime}}$ is the ancestor of $\frac{y}{x}$ that is closer to $\frac{y}{x}$ (in terms of graph-theoretic distance in $\mathcal{S B}$ ) and that has $\frac{y}{x}$ in its right subtree. The right child of $\frac{y}{x}$ is the node $\frac{\left(y+y^{\prime \prime}\right)}{\left(x+x^{\prime \prime}\right)}$, where $\frac{y^{\prime \prime}}{x^{\prime \prime}}$ is the ancestor of $\frac{y}{x}$ that is closer to $\frac{y}{x}$ and that has $\frac{y}{x}$ in its left subtree. The first level of $\mathcal{S B}$ is composed of node $\frac{1}{1}$. The $i$-th level of $\mathcal{S B}$ is composed of the children of the nodes of the $(i-1)$-th level of $\mathcal{S B}$.

\section{Appendix B: Proofs}

\section{Proofs from Section 3}

Lemma 2. For every $n \geq 3$ there exists an embedded planar graph $G_{\phi}$ with $3 n$ vertices and $3 n$ edges that does not admit any straight-line monotone drawing.

Proof. Let $G_{\phi}$ be the embedded planar graph consisting of a simple cycle $C=v_{1}, \ldots, v_{2 n}$ of length $2 n$ and of $n$ vertices $u_{1}, u_{3}, \ldots, u_{2 n-1}$ of degree 1 , called legs, incident to the 
vertices $v_{1}, v_{3}, \ldots, v_{2 n-1}$ of $C$ with odd indices, respectively. The embedding of $G_{\phi}$ is such that all the degree- 1 vertices are inside $C$, that is, are inside the unique internal face of $C$; see Fig. 5(a).

We prove that there exists no straight-line monotone drawing of $G_{\phi}$. Assume, for a contradiction, that such a drawing $\Gamma$ exists. Consider two arbitrary consecutive legs $u_{i-1}$ and $u_{i+1}$ of $G_{\phi}$; see Fig. 5(a). Since $\Gamma$ is monotone, there exists a monotone path between $u_{i-1}$ and $u_{i+1}$. This could be either the path $P_{i}^{1}=u_{i-1}, v_{i-1}, v_{i}, v_{i+1}, u_{i+1}$ or the path $P_{i}^{2}=u_{i-1}, v_{i-1}, v_{i-2}, \ldots, v_{1}, v_{2 n}, \ldots, v_{i+2}, v_{i+1}, u_{i+1}$.

We show that path $P_{i}^{2}$ can not be monotone. Refer to Fig. 5(b).

Assume for a contradiction that $P_{i}^{2}$ is monotone. Then, by Property 2 , edges $\left(v_{i-1}, u_{i-1}\right)$ and $\left(v_{i+1}, u_{i+1}\right)$ diverge, as $u_{i-1}$ and $u_{i+1}$ are leaves of $P_{i}^{2}$. Hence, in order to connect $v_{i-1}$ to $v_{i+1}$ with a polyline while keeping $u_{i-1}$ and $u_{i+1}$ inside the polygon representing $C$, at least three straight-line segments are necessary. However, only two edges, namely $\left(v_{i-1}, v_{i}\right)$ and $\left(v_{i}, v_{i+1}\right)$, lie between $v_{i-1}$ and $v_{i+1}$ in $C \backslash P_{i}^{2}$, which implies that at least one bend is needed in at least one of such edges, a contradiction.

Thus, for any arbitrary pair of consecutive legs $u_{i-1}$ and $u_{i+1}$ the monotonicity between them is provided by path $P_{i}^{1}$. However, we show that this leads to a contradiction.

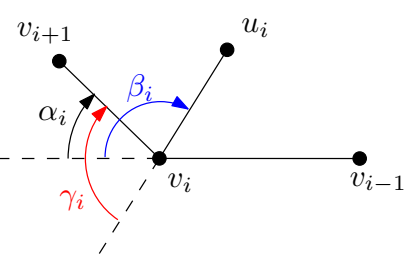

(a)

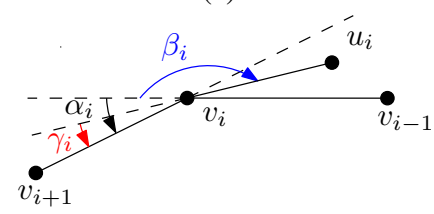

(c)

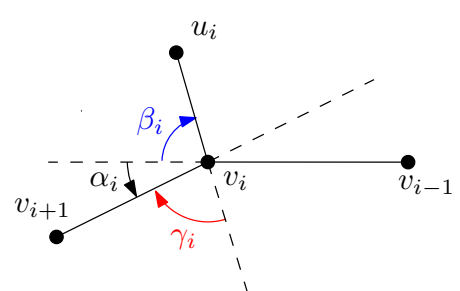

(b)

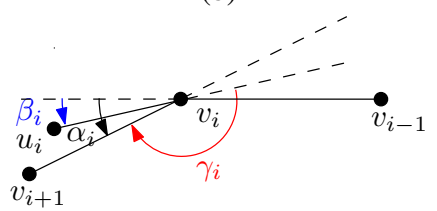

(d)

Fig. 9: The proof that equality $\beta_{i}+\gamma_{i}=\alpha_{i}+180$ holds for each $i=1,3, \ldots, 2 n-1$. (a) The internal angle at $v_{i}$ is convex. Equality $|\beta|+|\gamma|=180+|\alpha|$ holds and $\alpha, \beta, \gamma \geq 0$. (b) The internal angle at $v_{i}$ is reflex and the leg $u_{i}$ is between the half-line from $v_{i-1}$ through $v_{i}$ and the half-line from $v_{i+1}$ through $v_{i}$ in the circular ordering around $v_{i}$. Equality $|\beta|+|\gamma|=180-|\alpha|$ holds, and $\alpha \leq 0 ; \beta, \gamma \geq 0$. (c) The internal angle at $v_{i}$ is reflex and the leg $u_{i}$ is between the half-line from $v_{i+1}$ through $v_{i}$ and edge $\left(v_{i-1}, v_{i}\right)$ in the circular ordering around $v_{i}$. Equality $|\beta|-|\gamma|=180-|\alpha|$ holds, and $\alpha, \gamma \leq 0 ; \beta \geq 0$. (d) The internal angle at $v_{i}$ is reflex and the leg $u_{i}$ is between the half-line from $v_{i-1}$ through $v_{i}$ and edge $\left(v_{i+1}, v_{i}\right)$ in the circular ordering around $v_{i}$. Equality $|\gamma|-|\beta|=180-|\alpha|$ holds, and $\alpha, \beta \leq 0 ; \gamma \geq 0$.

Let $\alpha_{i}, i=1, \ldots, 2 n$, be the turn angle from edge $\left(v_{i-1}, v_{i}\right)$ to edge $\left(v_{i}, v_{i+1}\right)$, where the indices are taken modulo $2 n$. Let also $\beta_{i}$ and $\gamma_{i}, i=1,3, \ldots, 2 n-1$ be the 
turn angles from $\left(v_{i-1}, v_{i}\right)$ to $\left(v_{i}, u_{i}\right)$, and from $\left(u_{i}, v_{i}\right)$ to $\left(v_{i}, v_{i+1}\right)$, respectively. Note that:

$$
\sum_{i=1}^{2 n} \alpha_{i}=2 \pi .
$$

Also, as shown in Fig. 9(a-d), regardless of whether the internal angle at $v_{i}$ is convex or reflex and of the position of the leg $u_{i}$, the following equation holds:

$$
\beta_{i}+\gamma_{i}=\alpha_{i}+\pi, i=1,3, \ldots, 2 n-1
$$

Further, as path $P_{i}^{1}$ from $u_{i-1}$ to $u_{i+1}$ through $v_{i}$ is monotone, we have that $\gamma_{i-1}+$ $\alpha_{i}+\beta_{i+1}<\pi$ holds for each $i=1,3, \ldots, 2 n-1$, where the indices are taken modulo $2 n$; see Fig. 10.

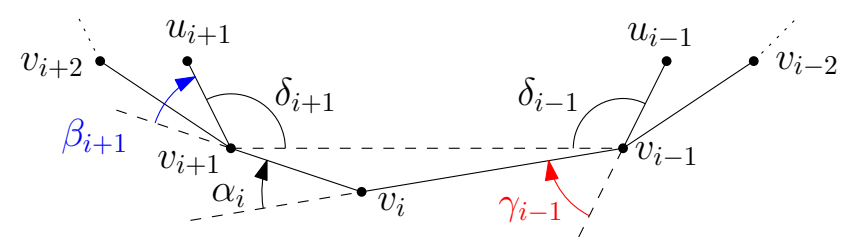

Fig. 10: $\delta_{i-1}+\delta_{i+1}+\gamma_{i-1}+\alpha_{i}+\beta_{i+1}=2 \pi$. As legs $u_{i-1}$ and $u_{i+1}$ diverge, $\delta_{i-1}+\delta_{i+1} \geq \pi$. Hence, $\gamma_{i-1}+\alpha_{i}+\beta_{i+1}<\pi$

By summing up these inequalities over $i=1,3, \ldots, 2 n-1$ we get: $\gamma_{1}+\alpha_{2}+\beta_{3}+$ $\gamma_{3}+\alpha_{4}+\beta_{5}+\cdots+\gamma_{2 n-1}+\alpha_{n}+\beta_{1}=\left(\beta_{1}+\gamma_{1}\right)+\alpha_{2}+\left(\beta_{3}+\gamma_{3}\right)+\alpha_{4}+\cdots+$ $\left(\beta_{2 n-1}+\gamma_{2 n-1}\right)+\alpha_{n}<n \pi$. Applying equation 2 , we get $\left(\alpha_{1}+\pi\right)+\alpha_{2}+\left(\alpha_{3}+\right.$ $\pi)+\alpha_{4}+\cdots+\left(\alpha_{2 n-1}+\pi\right)+\alpha_{n}=\sum_{i=1}^{2 n} \alpha_{i}+n \pi<n \pi$. By equation 1, we get $(n+2) \pi<n \pi$, a contradiction.

Lemma 3. For every odd $n \geq 9$ there exists an embedded planar graph $G_{\phi}$ with $n$ vertices and $\frac{3}{2}(n-1)$ edges such that every monotone drawing of $G_{\phi}$ has at least $\frac{n-3}{6}$ edges with at least two bends and thus at least $\frac{n-3}{3}$ bends in total.

Proof. Consider an odd integer $n \geq 9$. We construct $G_{\phi}$ iteratively. Let $G_{\phi}^{1}$ be a triangle graph. Graph $G_{\phi}^{i}$ is constructed from $G_{\phi}^{i-1}$ as follows. Initialize $G_{\phi}^{i}=G_{\phi}^{i-1}$. Let $(u, v, w)$ be a triangular internal face of $G_{\phi}^{i}$. Add 6 new vertices $u_{1}, u_{2}, v_{1}, v_{2}, w_{1}, w_{2}$ and 9 new edges $\left(u, u_{1}\right),\left(u, u_{2}\right),\left(u_{1}, u_{2}\right),\left(v, v_{1}\right),\left(v, v_{2}\right),\left(v_{1}, v_{2}\right),\left(w, w_{1}\right),\left(w, w_{2}\right)$, $\left(w_{1}, w_{2}\right)$ to $G_{\phi}^{i}$ in such a way that all the new vertices are inside $(u, v, w)$. Note that the $n$-vertex graph $G_{\phi}^{i}$ is planar and has $\frac{3}{2}(n-1)$ edges; see Figure 6(a).

Then, we prove that any monotone drawing of $G_{\phi}$ has at least $\frac{n-3}{6}$ edges with at least two bends.

Let $G_{\phi}^{t}$ be a subgraph of $G_{\phi}$ induced by a triangle $(u, v, z)$ of $G_{\phi}$ and by all the vertices drawn inside it (see Figure 6(b)). Let $\Gamma$ be a drawing of $G_{\phi}$ and let $\Gamma^{t}$ be a 
drawing of $G_{\phi}^{t}$ that coincides with $\Gamma$ restricted to the edges of $G_{\phi}^{t}$. We observe the following.

Observation 1 If $\Gamma^{t}$ is not a monotone drawing, then $\Gamma$ is not a monotone drawing.

In order to prove the observation, consider two vertices $a$ and $b$ of $G_{\phi}^{t}$ that are not connected by any monotone path in $\Gamma^{t}$. Assume, for a contradiction, that $\Gamma$ is monotone. Then, $a$ and $b$ are connected by a monotone path $P$ in $\Gamma$. As $P$ does not lie entirely in $G_{\phi}^{t}$, it passes twice through a cut vertex $c$ which is a common vertex of $G_{\phi}^{t}$ and $G_{\phi}$. Thus, the path $P_{t}$ obtained from $P$ by removing the part between the two occurrences of $c$ is a monotone path between $a$ and $b$ only composed of edges of $G_{\phi}^{t}$, a contradiction.

Observation 2 In any monotone drawing of $G_{\phi}^{t}$, at least one of the edges $(u, v),(v, w)$, $(w, u)$ has two bends.

In order to prove the observation, consider the subgraph $G_{\phi}^{h}$ of $G_{\phi}^{t}$ induced by vertices $u, v, w, u_{1}, v_{1}$, and $w_{1}$; see Fig. 6(c). Note that every monotone drawing of $G_{\phi}^{t}$ restricted to the edges of $G_{\phi}^{h}$ is a monotone drawing of $G_{\phi}^{h}$. Thus, if there exists a monotone drawing of $G_{\phi}^{t}$ such that none of the edges $(u, v),(v, w),(w, u)$ has two bends, then $G_{\phi}^{h}$ also has such a monotone drawing. However, we show that $G_{\phi}^{h}$ does not admit any of such drawings.

Let $G_{\phi}^{s}$ be a 1-subdivision of $G_{\phi}^{h}$. Note that, $G_{\phi}^{s}$ satisfies the preconditions of Lemma 2, that is, it is composed of a cycle plus a set of internal legs connected to every second vertex of the cycle, and hence it does not admit any straight-line monotone drawing. Thus, $G_{\phi}^{h}$ does not admit any monotone drawing with curve complexity 1 , as bends on the edges of $G_{\phi}^{h}$ correspond to the subdivision vertices in $G_{\phi}^{s}$.

The statement follows from the two observations. Namely, Observation 1 implies that the drawing of every subgraph defined as $G_{\phi}^{t}$ is monotone, and Observation $2 \mathrm{im}$ plies that in any monotone drawing of such a subgraph one of the edges of the outer triangle of $G_{\phi}^{t}$ has two bends. As the number of different triangles that contain a subgraph $G_{\phi}^{t}$ in their interior is $\frac{n-3}{6}$, any monotone drawing of $G_{\phi}$ has at least $\frac{n-3}{6}$ edges with two bends, and thus $\frac{n-3}{3}$ bends in total.

\section{Proof from Section 4.2}

Theorem 3. Every biconnected embedded planar graph admits a straight-line monotone drawing, which can be computed in linear time.

Proof. First, recall that the algorithm to compute a straight-line monotone drawing of any biconnected planar graph $G$ in the variable embedding setting [1] inductively constructs a drawing of $G$ by means of a bottom-up visit of the SPQR-tree of $G$, as follows.

When a component $\mu$ with child components $\mu_{1}, \ldots, \mu_{k}$ is visited, a drawing of the pertinent of $\mu$ satisfying certain monotonicity properties is constructed by composing the drawings of $\mu_{1}, \ldots, \mu_{k}$ which, by induction, satisfy the same properties. The composition is based on whether $\mu$ is an $S_{-}$, a $P_{-}$, a $Q-$, or an $R$-node. Note that, the 
properties satisfied by the drawing of $\mu$ ensure the monotonicity of the final drawing of $G$. Further, recall that one of these properties requires the drawing of the pertinent of $\mu$ to be entirely contained inside a boomerang $\operatorname{boom}(\mu)$.

Our algorithm for biconnected embedded planar graphs works in a similar fashion, except that, the pertinent graph of $\mu$ might be drawn either inside a boomerang $\operatorname{boom}(\mu)$ or inside a diamond $\operatorname{diam}(\mu)$, depending on the node-type of $\mu$. Namely, if $\mu$ is a $Q-$, an $S$-, or an $R$-node, then it is drawn inside a boomerang, while if it is a $P$-node then it is drawn inside a diamond. Hence, the drawing algorithm has to be modified in order to deal with the case in which the drawings of $\mu_{1}, \ldots, \mu_{k}$ are inside diamonds. Note that, this can happen only when $\mu$ is $Q-$, an $S$ - or an $R$-node, as two $P$-nodes cannot be adjacent in the SPQR-tree of $G$.

If $\mu$ is a Q-node, then draw an edge between the points $p_{N}(\mu)$ and $p_{S}(\mu)$ of boom $(\mu)$.

If $\mu$ is an S-node, then apply the same algorithm as for the variable embedding case, except for the fact that some child components of $\mu$, namely the parallel child components, might be drawn inside a diamond instead of inside a boomerang; see Fig. 11(a). However, as $p_{N}\left(\mu_{i}\right)$ and $p_{S}\left(\mu_{i}\right)$, for each $i=1, \ldots, k-1$, are placed on the bisector line of $p_{W}(\mu) \widehat{p_{N}(\mu)} p_{E}(\mu)$, the diamond $\operatorname{diam}\left(\mu_{i}\right)$ of any child parallel component $\mu_{i}$ lies entirely inside $\operatorname{boom}(\mu)$, still satisfying all the inductive properties. The same holds for $\mu_{k}$, as $p_{N}\left(\mu_{k}\right)$ and $p_{S}\left(\mu_{k}\right)$ are on the bisector line of $p_{W}(\mu) \widehat{p_{S}(\mu)} p_{E}(\mu)$.

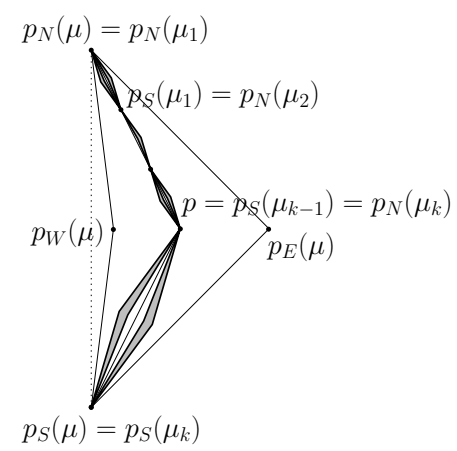

(a)

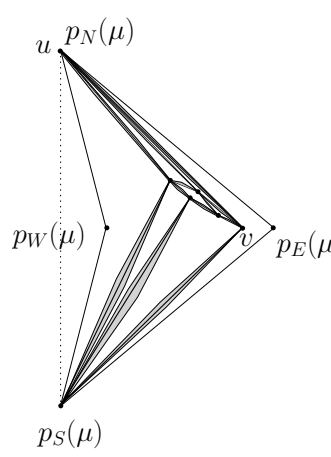

(b)

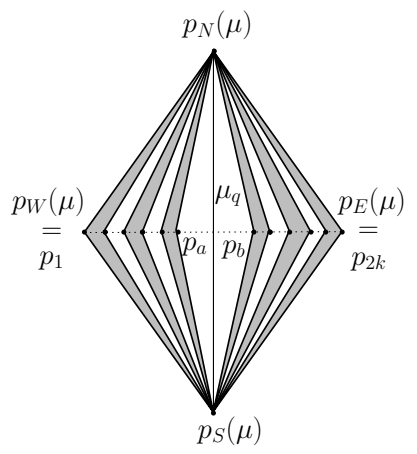

(c)

Fig. 11: Construction of a drawing of $\mu$ respecting the inductive hypothesis. (a) $\mu$ is an $S$-node. (b) $\mu$ is an $R$-node. (c) $\mu$ is a $P$-node.

More formally, let $p$ be the intersection point between segment $\overline{p_{W}(\mu) p_{E}(\mu)}$ and the bisector line of $p_{W}(\mu) \widehat{p_{N}(\mu)} p_{E}(\mu)$. Consider $k$ equidistant points $p_{1}, \ldots, p_{k}$ on segment $\overline{p_{N}(\mu) p}$ such that $p_{1}=p_{N}(\mu)$ and $p_{k}=p$. For each $\mu_{i}$, with $i=1, \ldots, k-$ 1 , consider a boomerang $\operatorname{boom}\left(\mu_{i}\right)=\left(\left(p_{N}\left(\mu_{i}\right), p_{E}\left(\mu_{i}\right), p_{S}\left(\mu_{i}\right), p_{W}\left(\mu_{i}\right)\right)\right.$ such that $p_{N}\left(\mu_{i}\right)=p_{i}, p_{S}\left(\mu_{i}\right)=p_{i+1}$, and $p_{E}\left(\mu_{i}\right)$ and $p_{W}\left(\mu_{i}\right)$ determine $\beta_{\mu_{i}}+2 \alpha_{\mu_{i}}<\frac{\alpha_{\mu}}{2}$. If $\mu_{i}$ is a $P$-node, consider a diamond $\operatorname{diam}\left(\mu_{i}\right)$ composed of two mirroring copies of $\operatorname{boom}\left(\mu_{i}\right)$. Then, apply the inductive algorithm to $\mu_{i}$ and either boom $\left(\mu_{i}\right)$ or $\operatorname{diam}\left(\mu_{i}\right)$. Also, consider a boomerang boom $\left(\mu_{k}\right)=\left(p_{N}\left(\mu_{k}\right), p_{E}\left(\mu_{k}\right), p_{S}\left(\mu_{k}\right), p_{W}\left(\mu_{k}\right)\right)$ such 
that $p_{N}\left(\mu_{k}\right)=p, p_{S}\left(\mu_{k}\right)=p_{S}(\mu)$, and $p_{E}\left(\mu_{k}\right)$ and $p_{W}\left(\mu_{k}\right)$ determine $\beta_{\mu_{k}}+2 \alpha_{\mu_{k}}<$ $\frac{\alpha_{\mu}}{2}$. If $\mu_{k}$ is a $P$-node, consider a diamond $\operatorname{diam}\left(\mu_{k}\right)$ composed of two mirroring copies of boom $\left(\mu_{k}\right)$. Then, apply the inductive algorithm to $\mu_{k}$ and to either $\operatorname{boom}\left(\mu_{k}\right)$ or $\operatorname{diam}\left(\mu_{k}\right)$.

If $\mu$ is an $R$-node, again, the algorithm is almost the same as in the variable embedding case; see Fig. 11(b). Indeed, the drawing of the skeleton of $\mu$ produced by the two algorithms is exactly the same. We describe how to place the boomerangs and the diamonds containing the inductively constructed drawings of the child components. Note that, two diamonds replacing two virtual edges that are consecutive in the circular order of the virtual edges around a vertex fall in the same face with one of their sides, which might not happen if the virtual edges were replaced by boomerangs. However, as in the variable-embedding algorithm no assumption is made about the face in which a boomerang replacing a virtual edge is placed, this situation is actually already handled in such an algorithm. Since the variable embedding algorithm ensures that the inductive properties are satisfied, the same holds for the fixed embedding algorithm.

The only problem occurs when the child component $\mu_{i}$ corresponding to the virtual edge connecting the vertices $u$ and $v$ placed on $p_{N}(\mu)$ and on $p_{E}(\mu)$, respectively, is a $P$-node and hence needs to be drawn inside a diamond $\operatorname{diam}\left(\mu_{i}\right)$. This would imply that $\operatorname{diam}\left(\mu_{i}\right)$ be not completely inside $\operatorname{boom}(\mu)$, which would violate the inductive hypothesis. However, in order to deal with this problem, it suffices to draw the skeleton of $\mu$ in such a way that $v$ is placed not exactly on $p_{E}(\mu)$, but on a point inside $\operatorname{boom}(\mu)$ that is close to it and that is still visible from $p_{S}(\mu)$. In this way, virtual edge $(u, v)$ does not lie on the boundary of $\operatorname{boom}(\mu)$ and both the sides of $\operatorname{diam}\left(\mu_{i}\right)$ can be drawn inside $\operatorname{boom}(\mu)$.

If $\mu$ is a P-node, recall that $\mu$ has to be drawn inside a diamond $\operatorname{diam}(\mu)$, while all its child components $\mu_{i}$, with $i=1, \ldots, k$, can be inductively drawn inside boomerangs boom $\left(\mu_{i}\right)$, as none of them can be a $P$-node. Further, note that at most one child component $\mu_{q}$ of $\mu$ can be a $Q$-node representing an edge between the poles of $\mu$. Draw such an edge, if any, as a straight-line segment between $p_{N}(\mu)$ and $p_{S}(\mu)$. Refer to Fig. 11(c). Then, in order to respect the given embedding around the poles of $\mu$, the child components $\mu_{1}, \ldots, \mu_{q-1}$ are drawn inside boomerangs that are contained into the left side of $\operatorname{diam}(\mu)$, while the child components $\mu_{q+1}, \ldots, \mu_{k}$ are drawn inside boomerangs that are contained into the right side of $\operatorname{diam}(\mu)$.

More formally, consider two internal points $p_{a}$ and $p_{b}$ of segment $\overline{p_{W}(\mu) p_{E}(\mu)}$ such that $p_{a}$ is to the left of segment $\overline{p_{N}(\mu) p_{S}(\mu)}$ and $p_{b}$ is to the right of $\overline{p_{N}(\mu) p_{S}(\mu)}$. Further, consider $2(q-1)$ points $p_{1}, \ldots, p_{2(q-1)}$ on segment $\overline{p_{W}(\mu) p_{a}}$ such that $p_{1}=$ $p_{W}(\mu), p_{2(q-1)}=p_{a}$, and $p_{i}{\widehat{p_{N}(\mu) p_{i+1}}}_{i(q-1)-1}$, for each $i=1, \ldots, 2(q-1)-1$. For each $\mu_{i}$, with $i=1, \ldots, q-1$, consider a boomerang boom $\left(\mu_{i}\right)=\left(p_{N}\left(\mu_{i}\right), p_{E}\left(\mu_{i}\right)\right.$, $\left.p_{S}\left(\mu_{i}\right), p_{W}\left(\mu_{i}\right)\right)$ such that $p_{N}\left(\mu_{i}\right)=p_{N}(\mu), p_{S}\left(\mu_{i}\right)=p_{S}(\mu), p_{E}\left(\mu_{i}\right)=p_{2 i-1}$, and $p_{W}\left(\mu_{i}\right)=p_{2 i}$. Apply the inductive algorithm to $\mu_{i}$ and boom $\left(\mu_{i}\right)$. Finally, consider $2(k-q)$ points $p_{q+1}, \ldots, p_{2 k}$ on segment $p_{b} \overline{p_{E}(\mu)}$ such that $p_{q+1}=p_{b}, p_{2 k}=p_{E}(\mu)$, and $p_{i} \widehat{p}_{N}(\mu) p_{i+1}=\frac{\alpha_{\mu}}{2(k-q)-1}$, for each $i=q+1, \ldots, 2 k-1$. For each $\mu_{i}$, consider a boomerang boom $\left(\mu_{i}\right)=\left(p_{N}\left(\mu_{i}\right), p_{E}\left(\mu_{i}\right), p_{S}\left(\mu_{i}\right), p_{W}\left(\mu_{i}\right)\right)$ such that $p_{N}\left(\mu_{i}\right)=$ $p_{N}(\mu), p_{S}\left(\mu_{i}\right)=p_{S}(\mu), p_{W}\left(\mu_{i}\right)=p_{2 i-1}$, and $p_{E}\left(\mu_{i}\right)=p_{2 i}$. Apply the inductive algorithm to $\mu_{i}$ and $\operatorname{boom}\left(\mu_{i}\right)$. 
Applying the same arguments as in [1], the statement follows. 